\title{
Representación de estructuras argumentativas mediante el análisis de redes sociales
}

\author{
J oel Martí - Departament de Sociología- UAB ${ }^{1}$
}

\section{Resumen}

Bajo el nombre de Análisis Reticular del Discurso (ARD) podemos englobar un conjunto muy amplio y diverso de técnicas que pretenden representar los discursos como una red de significados. La idea es bien sencilla: las palabras, acciones, proposiciones... son nodos de un entramado lingüístico que las relaciona, y que puede ser estudiado mediante el análisis de redes sociales (Lozares y otros, 2003). El artículo aborda una aproximación al ARD desde la teoría de la argumentación en la lengua (Anscombre y Ducrot, 1983), que introduce la noción relacional de topos (plural, topoi). Es posible representar la superposición de todos los topoi identificados en una red discursiva que esquematiza sistemas cognitivo-ideológicos, potencial que contemplan ya los mismos autores de la teoría, aunque no llegan a desarrollar. El texto se presenta una propuesta metodológica en este sentido, y muestra los resultados de una aplicación empírica al estudio de los discursos sobre la formación y el empleo.

Palabras clave: Análisis Reticular del Discurso - Argumentación - Cognición Análisis de Redes Sociales.

\begin{abstract}
Reticular Analysis of Discourse (ARD) refers to a large range of techniques that intend to represent the discourse as a network of meanings. The idea is well simple: the words and/or propositions are nodes of a linguistic network that relates them, and that can be studied by Social Network Analysis (Lozares et al, 2003). This text focus on ARD from theory of argumentation within language (Anscombre and Ducrot, 1983), which introduces the relational notion of topos (plural, topoi). It is possible to represent the globally of all the topoi identifed in a discursive network that schematizes cognitive/ideological systems, a possibility already contemplated, by authors, although not developed. This text presents a methodological proposal in this direction, and samples the results from an empirical application at the study of the discourses about formation employment.
\end{abstract}

Key words: Network-based discourse analysis - Argumentation - Cognition Social network analysis.

\footnotetext{
${ }^{1}$ Enviar correspondencia a: \oel.Marti@uab.es
} 


\section{La aproximación socio-cognitiva al discurso y las estructuras discursivas como objeto de estudio}

En los últimos años, una perspectiva destacada en los estudios del discurso es el enfoque socio-cognitivo, en el que podemos situar en un lugar central los trabajos de Teun Van Dijk (1995, 1998). La idea básica de esta aproximación es la necesidad de considerar una interfaz entre un nivel macro del lenguaje (las ideologías) y un nivel micro (los discursos): la cognición social. Esta propuesta es especialmente clarificadora en un contexto en el que nociones como las de discurso, creencias o ideología se usan recurrentemente de forma confusa y polisémica, frecuentemente sin explicitarlas, y restándoles, por lo tanto, su potencial explicativo. Concretamente, y con relación a los objetivos de este trabajo, la introducción de una perspectiva socio-cognitiva permite distinguir el discurso como proceso/acto comunicativo, entendido como la manifestación específica de determinadas creencias en el marco de una situación de interacción concreta, de un "espacio" de creencias socialmente construidas, abstractas y relativamente estables en el tiempo y, por lo tanto, diferenciar lo que sería la dimensión más situacional y dinámica de una enunciación (objeto de estudio de la pragmática) de aquella otra dimensión en la que se centran frecuentemente las ciencias sociales: los posicionamientos y cosmovisiones que hay "más allá" de una producción discursiva concreta ${ }^{2}$.

Esta clarificación conceptual permite plantear mejor estrategias metodológicas de análisis del discurso: cuando se estudia la práctica discursiva en si misma, se necesitarán técnicas para el análisis de procesos (como las que se han desarrollado ampliamente en análisis conversacional), que muestren cómo la relación de poder condiciona/construye la dinámica de la interacción; en cambio, cuando se estudian las estructuras discursivas (las creencias e ideologías sobre las que se apoyan estas prácticas discursivas), se deberán utilizar fundamentalmente técnicas estructurales que muestren las reglas que guían la producción discursiva (la red de creencias que le da soporte) y vincular estas reglas con el proceso social estudiado y su estructura de poder/dominación. El desarrollo metodológico aquí presentado se centra en esta segunda dimensión del discurso.

\footnotetext{
2 Más allá de los objetivos inmediatos de este trabajo, otras distinciones conceptuales podrían hacerse. Por lo que respecta al discurso, puede referirse a la práctica comunicativa en si, al producto de esta práctica, o incluso al producto de un análisis del mismo. Por lo que respecta a las creencias y a las ideologías, entendiendo las segundas como una propiedad de las primeras, desde el momento en que la reproducción de determinadas creencias en el discurso contribuye a la producción, reproducción o transformación de relaciones de poder (Van Dijk, 1998).
} 
El estudio de las creencias y, particularmente, su "forma" o representación es un aspecto relativamente poco trabajado en sociología y disciplinas afines con las únicas excepciones, seguramente, de los modelos estructurales de Barthes, Greimas o Lévi-Strauss y las aplicaciones de modelos cognitivos que en la sociología han hecho Goffman (1974) y, evidentemente, Cicourel (por ejemplo, Cicourel y Carley 1990) ${ }^{3}$. Una perspectiva que sí que ha trabajado plenamente en este campo a lo largo de los últimos años ha sido la cognitiva, especialmente en su corriente norteamericana que, en contraste con la teoría de las representaciones sociales, de origen europeo y de orientación más teórica, se caracteriza por una dirección mucho más empírica.

Distinguiré, basándome en Searle (1984), dos programas de investigación en ciencia cognitiva: el "programa fuerte" y el "programa débil"; la primera versión es la más 'asocial' del cognitivismo y, por tanto, la de menor interés en sociología, mientras que la segunda introduce elementos pertinentes para el análisis del discurso desde las ciencias sociales y que han sido aplicados en campos como la antropología (Holland y Quinn 1997) o la misma aproximación socio cognitiva al discurso de Van Dijk (1998). Ambas líneas comparten el principio de que la actividad mental consiste en el procesamiento de información simbólica, aunque divergen en cuanto a su concreción. El "programa fuerte" está protagonizado por la inteligencia artificial y parte de la base de que el procesamiento mental de la información se basa en procedimientos lógicos y lineales. Para el "programa débil", en cambio, el pensamiento humano es más complejo e impredecible: se considera la capacidad humana para reflexionar, modificar o actuar contingentemente sobre estas lógicas, y se considera también que la incorporación de nueva información no implica la necesidad de tener que ordenarla globalmente cada vez (Cicourel 1989, 40).

La representación de esta segunda forma de pensamiento se ha englobado generalmente bajo el concepto de 'esquemas' que, aunque se han desarrollado de formas diversas, vienen a corresponder al uso cotidiano del término (por ejemplo, cuando decimos "esto que has hecho me ha roto los esquemas") ${ }^{4}$. Brewer y Nakamura (1984, 140-1) han definido los esquemas como estructuras mentales

\footnotetext{
${ }^{3}$ A este poco desarrollo ha contribuido, por otro lado, la generalización del análisis temático de contenido, al imponer al texto una categorización previamente establecida que lo segmenta y lo reconstruye sin establecer ningún diálogo con él, sino únicamente a partir del marco interpretativo del analista.

${ }^{4}$ La noción de 'esquema' es introducida por Kant en Crítica de la razón pura como "una regla de síntesis de la imaginación", y se define como disposiciones innatas de la mente que permiten integrar y ordenar datos procedentes del mundo exterior (Puente 1998, 399-400).
} 
que contienen conocimiento abstracto. Son modulares (diferentes dominios cognitivos tienen esquemas con diferentes características estructurales) y se adaptan a las situaciones concretas como modelos de situación.

Una de las concreciones operativas más clásicas de los esquemas han sido los guiones (scripts) propuestos en 1977 por Schank y Abelson (1977) y revisados posteriormente (Schank 1982). Los guiones son secuencias de acciones estereotipadas respecto a determinados tipos de actividades que responden a finalidades (planes) orientadas a una tarea específica y que contienen informaciones sobre el tipo de marco (setting) en el cual se desarrolla la interacción, sobre los/las participantes que intervienen y sobre los roles y posiciones que ocupan. Abelson los define como:

Secuencia de acontecimientos esperados por el individuo, que lo implican a él mismo como participante o como observador. (Abelson 1976, 33)

Así, los autores muestran cómo ir a un restaurante, por ejemplo, supone seguir un conjunto de acciones que se asumen por defecto (entrar, sentarse, pedir, comer, pagar, etcétera). Estos detalles o guiones, socialmente compartidos a partir de la experiencia cotidiana, son, para Schank y Abelson, los que permiten crear expectativas o predicciones sobre una interacción y por tanto planificar acciones, así como inferir informaciones que "faltan" en los discursos y comprenderlos globalmente ${ }^{5}$.

Los guiones y otros tipos de esquemas han sido aplicados en ciencias sociales para representar creencias socialmente construidas. Para Goffman (1974), los marcos de actividad son esquemas de interpretación socialmente construidos mediante los cuales uno/a da sentido a una interacción: en una determinada situación y a partir de determinados indicios que de ella se desprenden, los/las participantes "buscan" inconscientemente esquemas que contengan pautas de actividad que se correspondan. En otro sentido, Linde (1997) propone un tipo de modelo cognitivo que llama 'sistema explicativo' como forma de representación social de la propia trayectoria, a medio camino entre la vida cotidiana y los sistemas teóricos, que permiten dar consistencia al discurso. Linde restringe los sistemas explicativos a aquel tipo de explicaciones que aluden a algún tipo de teoría "experta" socialmente

\footnotetext{
${ }^{5}$ Por ejemplo, es innecesario decir que un cliente en un restaurante paga la factura, porque el guión de esta situación lo presupone; en cambio, el enunciado "Juan fue al parque, se vistió y se fue" es pragmáticamente inaccesible porque no hay ningún guión compartido que incluya estas actividades (Schank y Abelson 1977, 54).
} 
compartida (por ejemplo, explicaciones psicoanalíticas, astrológicas o religiosas sobre los hechos), frente a aquellas explicaciones basadas en intenciones (Linde 1997, 351).

Un sistema explicativo es un sistema de creencias y relaciones entre creencias que configura un entorno en el cual una proposición puede ser tomada o no como causa de otra proposición. (Linde 1997, 343)

De esta forma, los sistemas explicativos pueden ser interpretados como "guiones sociales" que contienen sistemas de aseveraciones, declaraciones y de relaciones entre ellas que permiten que una proposición o expresión pertinente a su ámbito pueda implicar otra. $Y$, en el mismo sentido, pueden plantearse guiones de alcance más amplio con claras propiedades ideológicas que no pasen por situaciones con una secuencialidad tan local como el ejemplo del restaurante sino que abarquen trayectorias sociales entendidas globalmente, como por ejemplo los guiones de género o de clase.

\section{La contribución de la teoría de la argumentación. El topos como herramienta}

Desde la tradición estructural también se han desarrollado herramientas de análisis de las estructuras lingüísticas, aunque, como afirma Johnson-Laird (1990: 27), frecuentemente basadas en oposiciones binarias excesivamente simples como para representar muchos tipos de pensamiento. Pero desde esta misma tradición se ha desarrollado también la teoría de la argumentación (Anscombre y Ducrot, 1983), que, a pesar de las distancias teóricas y epistemológicas existentes con el enfoque tratado en el apartado anterior, presenta puntos de encuentro de notable interés al articular el uso del lenguaje en situación (pragmática) con su construcción histórica (estructural).

El concepto central de la teoría de la argumentación es el topos (pl. topoi). Para Anscombre y Ducrot, un topos es una relación discursiva entre dos proposiciones interdependientes, $\mathrm{P}$ y $\mathrm{Q}$, que puede ser vista como una estructura argumentoconclusión que da soporte a un enunciado (por ejemplo, si alguien dice "he encontrado trabajo porque tenía buenos contactos", desde la teoría de la argumentación se diría que este argumento es comprensible no tanto por la presencia de la preposición "porque", sino debido al hecho de que existe un topos, una creencia o lógica socialmente compartida, de que tener buenos contactos facilita la inserción laboral). Para Anscombre y Ducrot, el uso de topoi tiene como 
finalidad la construcción de representaciones ideológicas que dan legitimidad a un enunciado en el marco de una interacción particular.

Como se muestra en el cuadro adjunto, sobre cada esquema tópico (relación entre dos proposiciones $\mathrm{P}$ y $\mathrm{Q}$ ) se pueden establecer dos topoi, uno en positivo y el otro en negativo, y cada uno de ellos se puede manifestar en dos formas tópicas distintas (en el ejemplo, si asumimos que P es "tener dinero" y Q "ser feliz", los dos topoi son pragmáticamente aceptables -materialismo en positivo, y voto de pobreza en negativo).

\begin{tabular}{|c|c|c|}
\hline Esquema tópico & Topos & Formas tópicas \\
\hline$<$ Dinero Felicidad $>$ & $\begin{array}{c}\text { DINERO -> FELICIDAD } \\
\text { (materialismo) } \\
\text { DINERO -> NO FELICIDAD } \\
\text { (voto de pobreza) }\end{array}$ & $\begin{array}{l}<+p,+q> \\
\text { Más dinero, más felicidad } \\
<-p,-q> \\
\text { Menos dinero, menos felicidad } \\
<-p,+q> \\
\text { Menos dinero, más felicidad } \\
<+p,-q> \\
\text { Más dinero, menos felicidad }\end{array}$ \\
\hline
\end{tabular}

I lustración 1. Esquema tópico <Dinero Felicidad>

Hay, evidentemente, una analogía entre los topoi y los implícitos trabajados en las tradiciones anglosajonas de la pragmática, especialmente con la noción de implícito conversacional de Grice (1975). Sin embargo, mientras que en la tradición anglosajona los implícitos se derivan del significado global del acto comunicativo en concreto (al nivel del discurso), los topoi se plantean como un principio previo a la interacción (al nivel de las creencias sociales). Es justamente a partir del discurso que estas creencias se evocan:

De manera general, el uso de estos topoi tiene como finalidad la construcción de representaciones ideológicas que no se declaran como tales, y su grande poder persuasivo procede del hecho que, si bien el locutor los convoca libremente, estos topoi se presentan como si fueran exteriores a él y, consecuentemente, objetivos. (Anscombre y Ducrot 1994, 249). 
El topos, pues, permite dar sentido a un discurso concreto, y constituye un mecanismo cognitivo básico a partir del cual interpretamos la realidad e interaccionamos. Para Anscombre y Ducrot, tiene tres propiedades fundamentales:

- En primer lugar, es un vínculo socialmente compartido, como mínimo por parte de algún grupo social.

- En segundo lugar, es una regla general (en el plano de la cognición social) que posibilita una argumentación particular, y por lo tanto tiene un carácter relativamente estable.

- En tercer lugar, es una regla gradual, de forma que se puede expresar como "a más A, más B".

A estas propiedades se puede añadir una cuarta, que de hecho contemplan ya los mismos autores de la teoría, aunque no llegan a desarrollar: un discurso se apoya en un conjunto de topoi que esquematiza sistemas cognitivos representables en una estructura o red de relaciones tópicas. Se trata de una representación que conserva, por lo tanto, la unidad del discurso sin fragmentarlo en categorías u otro tipo de clasificaciones preestablecidas, que sin duda son muy útiles como abstracción analítica, pero que no se corresponden con una compartimentación de ámbitos aislados y coherentes que las personas establezcan en sus vidas cotidianas o en sus discursos.

De estas bases teóricas se deriva, según Anscombre y Ducrot, que los topoi tienen como finalidad la construcción de representaciones cognitivas que dan legitimidad a un enunciado en el marco de una interacción particular $y$, por lo tanto, podemos mostrar los referentes ideológicos de la argumentación identificando qué topoi se utilizan y enfatizan en una argumentación, y poniéndolos en relación con la estructura/proceso social del que forman parte. Además, estas estructuras de topoi (o tópicas) no sólo permiten dar sentido al discurso, sino que también constituyen sistemas cognitivos mediante los cuales interpretamos el mundo y que, en última instancia, nos proporcionan esquemas y secuencias lógicas entre distintos acontecimientos; o, dicho de otra forma: la práctica social cotidiana se apoya en la forma en que distintos topoi se relacionen y articulen en estas estructuras discursivas. 
De lo que se ha venido diciendo hasta aquí, se pueden deducir ya los elementos fundamentales del enfoque metodológico que se presenta en este artículo: la identificación, en discursos, de relaciones -explícitas o implícitas, manifiestas o latentes- entre creencias sociales que, movilizadas en determinadas situaciones de interacción, pueden concretarse en guiones $y$, con ello, mostrar los esquemas cognitivos que dan soporte a determinadas prácticas sociales.

\section{Aplicación empírica del instrumento metodológico}

En este apartado se presenta una aplicación empírica de este instrumento al análisis de los discursos sobre la formación y el empleo que se mantienen desde trabajadores/as que ocupan empleos no cualificados, análisis desarrollado más ampliamente en Martí (2000). Corresponde a un trabajo realizado en el marco del Grup d'Estudis Sociològics sobre la Vida Quotidiana i el Treball de la Universidad Autónoma de Barcelona, en el que se profundizó en el Análisis Reticular del Discurso (Lozares y otros, 2003) mediante el desarrollo de distintos métodos e instrumentos de análisis.

Muy sintéticamente, el objetivo sustantivo del estudio realizado era analizar, en primer lugar, la incidencia que tenía entre los colectivos de trabajadores descualificados el topos dominante "a más formación, más y mejor empleo" y, en segundo lugar, se trataba de ver los efectos de este discurso en distintas trayectorias de clase y de género.

A partir de este planteamiento se analizaron ocho entrevistas procedentes de un estudio de caso (selección de entrevistados según edad, género y categoría laboral); se trataba de entrevistas centradas en las biografías, con especial incidencia en los aspectos formativos y laborales y también con algunas preguntas de tipo general referidas a percepciones de la formación.

El diseño de análisis aplicado, aun partiendo de un modelo previo y de criterios de interpretación vinculados a los objetivos sustantivos de la investigación (descartando, por lo tanto, la falsa ilusión de interpretación inductiva 'en el vacío'), se basó en la indexación de los topoi identificados en la interpetación del discurso de los sujetos entrevistados.

La sistematización de este trabajo de indexación se realizó mediante la creación de una sociomatriz de relaciones tópicas, positivas o negativas, entre pares de significados, dirigidas y valoradas mediante su frecuencia de aparición, y su posterior análisis mediante el álgebra de redes sociales. 


\section{a) Proceso de identificación de relaciones tópicas}

Antes de exponer los resultados se presenta un pequeño ejemplo de cómo se ha hecho el proceso de interpretación y análisis. El proceso de interpretación es básicamente cualitativo puesto que en última instancia el establecimiento de relaciones tópicas se basa en criterios contextuales (condicionantes y efectos del discurso). La cita que sigue corresponde a un fragmento de una de las entrevistas a una cajera de hipermercado 26 años:

...en qué medida te has sentido tú que participabas, en ese tipo de acontecimientos pues cuando te has puesto a trabajar, cuando has decidido casarte, cuando estabas estudiando. Y en qué medida te encontrabas tú como que... Hombre, yo cuando empecé a trabajar, pues con más libertad porque en mi casa, siempre era del instituto a mi casa, no. No tenía amigos, no salía apenas. Y te cambia mucho la vida porque ya salía, disponía de mi dinero que cambia. Luego enseguida ya..., empecé a salir con mi marido. Ya empecé a salir y me cambió la vida en ese año, a partir de los dieciocho años, para acá, sí, me ha cambiado la vida, es lo que más. Pero al principio era una cría, no tengo muchos recuerdos de..., hombre, la etapa del instituto que tampoco fue muy buena, porque no tenía amigos y no, lo pasé... Es que yo no soy muy comunicativa y lo pasé mal. Luego aquí ya me fui abriendo con la gente, también estaba de cara al público, te abres más, te haces más sociable. Pero poca cosa más. Es que no sé qué contarte...

Y este sería el grafo resultante de la interpretación (es decir, la plasmación gráfica de las relaciones tópicas establecidas en el proceso de interpretación). No es el único grafo posible, puesto que en última instancia la identificación de estas relaciones depende de los criterios interpretativos utilizados.

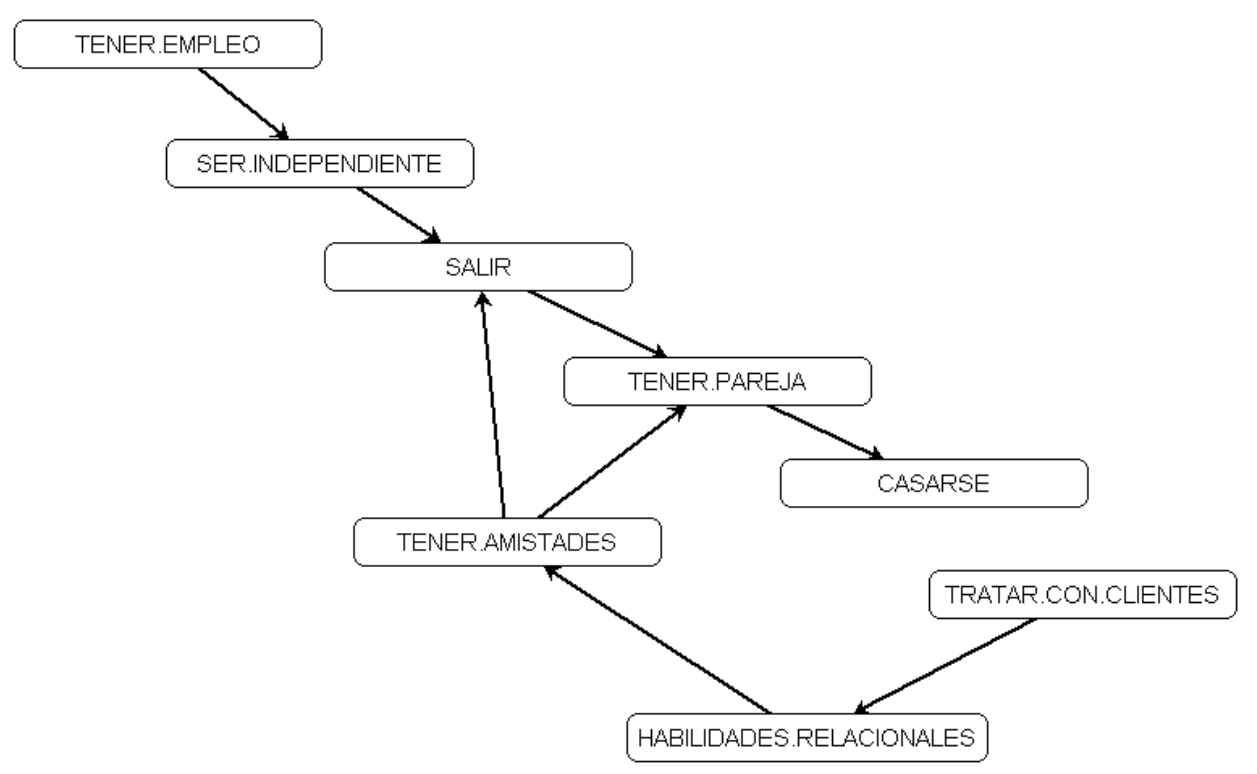

I lustración 2. Grafo 1 . Red de topoi 


\section{b) Criterios de análisis}

Más allá de la secuencialidad de la narración, la observación de esta estructura discursiva nos permite mostrar su forma a simple vista: este discurso parte del mundo laboral (gracias a éste se obtiene más independencia y se aprende a relacionarse con otros), fluye por el ocio (se sale, se conoce gente) y desemboca en el ámbito doméstico (una empieza a salir por ahí... y termina casándose). Ahora bien, la utilidad de este instrumento está no en fragmentos tan pequeños sino en la potencialidad de tener una red global de todo un texto, dónde estas relaciones se solapan con otras. Es por ello que para analizar estructuras más complejas se ha utilizado el álgebra de redes sociales, y concretamente tres indicadores básicos que definen distintas centralidades en la red discursiva (basadas en Freeman, 1979):

Grado nodal. Al ser un gráfico dirigido se puede distinguir entre grado nodal de salida y grado nodal de entrada; ambos indicadores muestran los elementos que aparecen en mayor medida en el discurso, sea como emisores o receptores.

Grado de accesibilidad. La accesibilidad de un nodo se refiere al grado en que éste puede acceder (o ser accedido, dependiendo que sea de entrada o de salida) a los otros nodos de la red ${ }^{6}$. Así, en el ejemplo anterior, los dos nodos que más directamente pueden acceder al resto de la red (esto es, accesibilidad de salida) son los relacionados con la actividad laboral y, por lo tanto, podríamos decir que la actividad laboral es el motor o lo generador de todo aquello que es narrado. En cuanto a la accesibilidad de entrada, el nodo "casarse" es el que más fácilmente es accedido por todo el resto de la red: se situaría aquí como un nodo finalista, como un punto de llegada de todo el discurso.

Grado de intermediación: Indica nodos estratégicos, porque se sitúan uniendo distintos subconjuntos, y en el ejemplo mencionado corresponden también al ámbito relacional, que se situaría aquí como mediador entre la actividad laboral y el ámbito doméstico.

Para cada una de las entrevistas analizadas se seleccionaron los nodos con valores más altos en cada indicador y se realizó una proyección gráfica de las relaciones existentes entre estos nodos, obteniéndose lo que denominamos "grafo de centralidades": se trata de un grafo con un número relativamente pequeño de

\footnotetext{
${ }^{6}$ Este indicador ha sido calculado a partir de la matriz de accesibilidad basada en la mínima amplitud (esto es, la amplitud de un lazo es igual a la amplitud del vínculo más débil).
} 
nodos, que aunque pueda parecer escueto con relación a todo el discurso, es un resultado que no viene impuesto por un sistema de categorías previo, sino que corresponde al núcleo argumentativo resultante de la identificación de topoi en el discurso y de su análisis mediante ARS.

\section{c) Síntesis de resultados}

Se muestran, a nivel descriptivo, dos grafos de centralidades, correspondientes a trayectorias de género clásicas (masculinas y femeninas). Son grafos muy reducidos respecto al trabajo original con la finalidad de facilitar la exposición, pero en ellos se ven los principales ejes de los discursos:

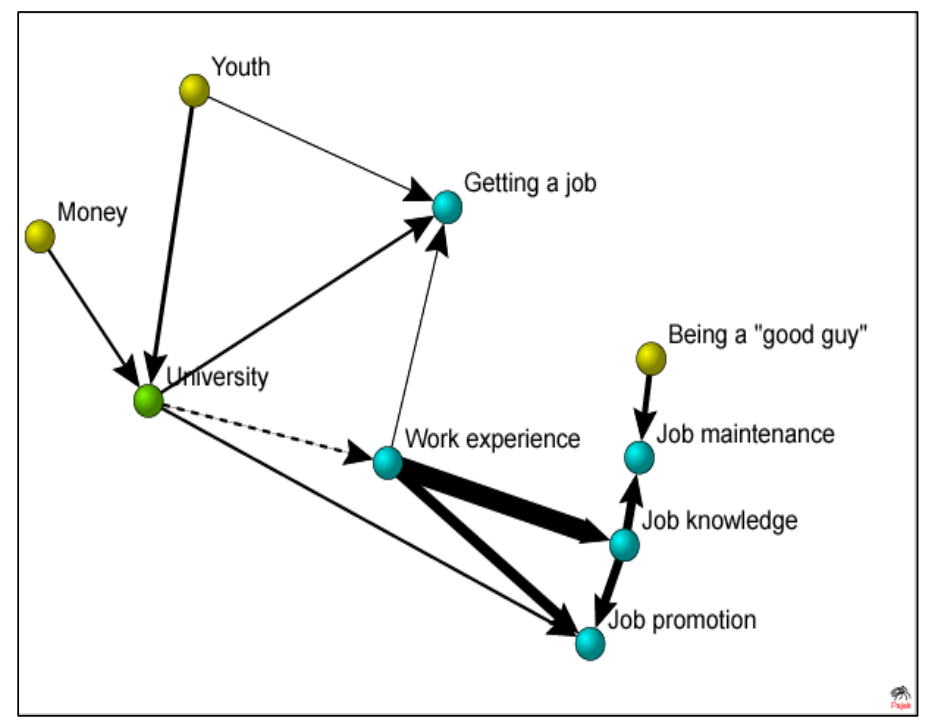

Color: Nodos azules: ámbito laboral; Nodos verdes: ámbito de formación; Nodos rojos, ámbito doméstico; Nodos amarillos: otros.

Grosor: Representa la intensidad de relación tópica, medida por su frecuencia de aparición Estilo: Las líneas discontínuas indican relación negativa.

\section{I lustración 3. Grafo 2. Trayectoria de centralidad productiva}

El Grafo 2 pertenece a un obrero industrial de 28 años, residiendo con sus padres pero con horizonte de emancipación cercano. Como principales elementos a destacar:

1) En primer lugar, el papel central que juegan los nodos referidos al ámbito laboral: tienen un alto grado nodal y una alta accesibilidad de entrada, lo que refleja la importancia dada a estos aspectos. Especialmente relevante en este contexto es la experiencia laboral para adquirir conocimientos, permanecer en la empresa y promocionar. 
2) Un segundo elemento que incide en el empleo son los estudios universitarios, que tiene una alta accesibilidad de salida y una alta intermediación. Sobre los estudios universitarios es importante destacar dos cosas:

- En primer lugar, que quién está hablando es un perfil de trabajador que en muchos casos ha hecho cursos de formación en la empresa pero que no tiene estudios universitarios y que, a la hora de destacar la incidencia de la formación en el empleo, destaca la que mayoritariamente no tiene: los estudios universitarios. Esto tiene su lógica, y es que el filtro que establece la empresa estudiada para el acceso a puestos cualificados es el de estudios universitarios, mientras que la formación interna en la empresa no garantiza, en términos generales, ni la permanencia ni la mejora laboral.

- En segundo lugar, la disyuntiva que se establece entre los estudios universitarios y la experiencia laboral (es decir, la idea de que "me encontré en un momento de mi vida en el que tenía que decidir si quería trabajar o quería estudiar").

3) Finalmente, es importante también destacar los factores a los que se atribuye la no posesión de una formación universitaria. Por una parte se recurre al topos del ciclo de vida (se estudia cuando se es joven, característica que el participante no se autoatribuye, a pesar de tener 28 años). Y por otra parte se introduce la idea de los condicionantes externos: la disponibilidad de dinero interviene en el hecho de poder o no poder estudiar. Estos dos nodos tiene la accesibilidad de salida más alta de toda la red, es decir, actúan como principios argumentativos que condicionan el resto del discurso $y$, por lo tanto, son los que explican en última instancia las posiciones laborales de las personas entrevistadas (siempre desde su punto de vista). Esto es importante sobretodo desde el momento que la persona que basa su discurso en este esquema tópico carece de estudios universitarios, y por lo tanto apunta a los factores mediante los cuales justifican la no posesión de los mismos. De esta forma, los estudios universitarios, que desde el punto de vista de estos discursos es importante en el mercado de trabajo, resultan una inversión demasiado costosa. Con lo cual, el uso del topos "a más formación, más empleo" se reproduce en el discurso cotidiano de trabajadores/as descualificados/as, pero no tiene una traducción práctica directa, sino que es básicamente un recurso discursivo para legitimar la propia descualificación laboral, hasta el punto que incluso un entrevistado lo expresa en una frase muy gráfica: "ahora ya no me puedo arreglar". 


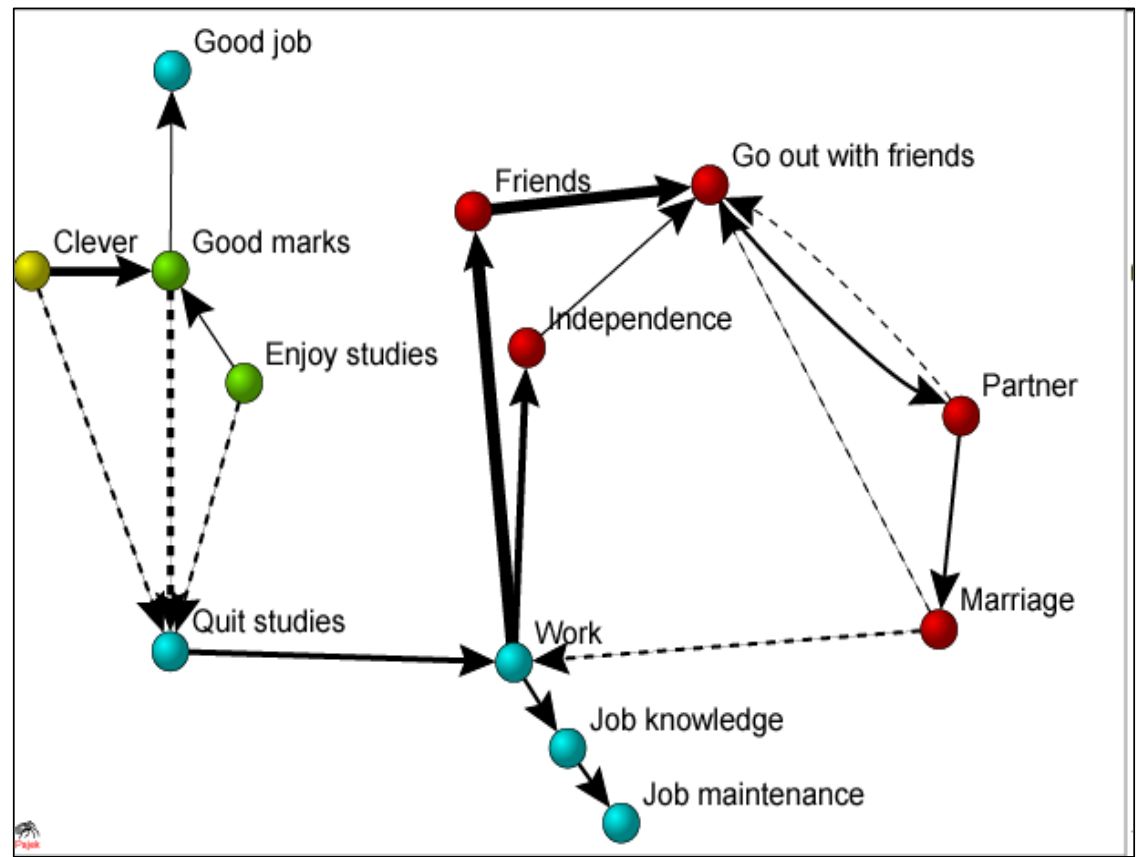

Color: Nodos azules: ámbito laboral; Nodos verdes: ámbito de formación; Nodos rojos, ámbito doméstico; Nodos amarillos: otros.

Grosor: Representa la intensidad de relación tópica, medida por su frecuencia de aparición Estilo: Las líneas discontínuas indican relación negativa.

\section{I lustración 4. Grafo 3. Trayectoria de doble presencia}

El Grafo 3 pertenece a una cajera de hipermercado (26 años y recientemente emancipada en pareja), y aquí el panorama cambia sensiblemente:

1) En primer lugar, y sobre todo, porque en la red discursiva la formación y el empleo pasan a jugar un papel relativamente periférico en comparación con el caso anterior; incluso ni tan sólo se habla de la promoción, sino sólo de la inserción laboral y de la permanencia en el empleo.

2) En cambio, los nodos referidos al ámbito doméstico-familiar (en rojo) juegan un papel central. Así, parece como si hubiera una secuenciación de la trayectoria en el sentido de una etapa para estudiar, una etapa para trabajar, una etapa para relacionarse y una etapa para consolidar el proyecto familiar y abandonar todo lo anterior (tener pareja y casarse implica dejar de salir y dejar el empleo). En estos casos, el valor atribuido a la formación es nulo porque, aunque se mantiene la importancia de la formación para tener un buen empleo, no existen proyectos profesionales futuros, y para la situación profesional presente no se necesita de tal formación. 
3) Y en tercer lugar, se acentúan respecto al tipo anterior las atribuciones internas en cuanto al hecho de tener más o menos formación: en estos casos, se habla de aspectos como el hecho de ser inteligente ("servir para los estudios"), o de "gustar o no gustar estudiar", ambos con la más alta accesibilidad de salida. De forma que la formación se acaba convirtiendo en algo ajeno o lejano y, al final, del mismo modo que en el caso anterior, se termina atribuyendo la posición formativa y laboral a una opción propia.

\section{Conclusiones: aportaciones y limitaciones del método y herramienta utilizados}

Podemos diferenciar las principales aportaciones del método utilizado en un doble plano, metodológico y tecnológico.

En el plano metodológico, y en primer lugar, permite abordar la interfaz cognitiva entre las creencias/ideologías (en abstracto y a nivel macro) y su manifestación como discurso en contextos de interacción particulares (micro). Evidentemente, ello no significa que las estructuras identificadas sean La Cognición (en mayúscula), sino un instrumento metodológico que nos permite visualizar cómo distintos 'esquemas' se estructuran y entrelazan en los discursos y en las trayectorias cotianas: es la superposición entre relaciones tópicas la que, en el marco de una situación de interacción o de una argumentación específica, dan lugar a discursos y prácticas sociales, tal y como apuntan Anscombre y Ducrot:

...la teoría de los topoi considera en efecto que 'bajo las palabras' se encuentran no objetos, sino guiones, o más bien, esquemas de guiones. (Anscombre y Ducrot 1994, 236, refiriéndose a los scripts)

En segundo lugar, desde un enfoque reticular no sólo se identifican patrones cognitivos estables y aplicados recurrentmente en las prácticas sociales, sino que, al mostrar los discursos en su globalidad, se identifican sus interacciones, contradicciones y sinergias; aspectos más difíciles de detectar en análisis cualitativos que fragmentan el texto en categorías o discursos temáticos (por ejemplo, el análisis de contenido). Retomando los supuestos teóricos presentados al principio, la plasmación gráfica de las relaciones tópicas muestra cómo se articulan distintas ideologías en una misma práctica social, de forma que, creencias que todo el mundo parece compartir (como el topos "a más formación, más y mejor empleo"), tienen traducciones muy distintas en el contexto de unas y otras estructuras discursivas, y por lo tanto tendrá unas concreciones prácticas muy 
distintas en unos y otros grupos sociales ${ }^{7}$. Un topos es, en última instancia, una "voz" (Bajtín 1981) o un "enunciador" (Anscombre y Ducrot 1994) que habla en el discurso y dialoga con los otros:

Lo que llamamos, en polifonía, 'el punto de vista de los enunciadores' no es más que la convocatoria de un topos mediante la aplicación de una forma tópica. (Anscombre y Ducrot 1994, 222)

La introducción de enfoques argumentativos y cognitivos en el análisis del discurso no debe suponer, sin embargo, omitir ni obviar la dimensión social del mismo, todo lo contrario: está presente en cada uno de los aspectos anteriores y en su interpretación: el planteamiento teórico previo y la identificación de la estructura social, grupos e intereses presentes es justamente lo que permite la comprensión e identificación de relaciones tópicas, y es lo que permite también introducir un nivel explicativo en el cual las estructuras cognitivas son el producto de las relaciones sociales y, a su vez, el vehículo a través del cual éstas se (re)producen.

En el plano tecnológico, la utilización del análisis de redes sociales se adecúa a este abordaje del nivel meso del lenguaje, además de permitir una objetivación del proceso analítico y la posibilidad de trabajar con grandes volúmenes de información discursiva, permitiendo realizar análisis a distintos niveles de concreción y generalización, así como centrar el interés en determinados subgrupos de nodos según ámbito o campo disursivos. Obviamente, el estatismo de las redes obliga a centrarse en el nivel estructural del discurso, y debe complementarse con otros enfoques desde el momento en que quieran abordarse otros niveles de análisis.

\section{Bibliografía}

Abelson, R. P. (1976). "Script processing in attitude formation and decision making". A: Cognition and social behavior, comp. per J. S. Carrol y J. W. Payne. Hillsdale, Nova Jersey: Erlbaum.

Anscombre, J. C.; Ducrot, O. (1983). L'argumentation dans la langue. Liège: Mardaga.

- Ducrot, O. (1994). La argumentación en la lengua, ed. rev. y aum. Madrid: Gredos.

\footnotetext{
7 La afirmación de que el topos dominante "a más formación, más empleo", o cualquier otro discurso hegemónico, es alienante, es extremadamente simplista en este contexto. Si las personas entrevistadas "obedecieran" alienadamente este discurso, entonces hubieran hecho las mil y una para lograr estudiar una carrera, pero no han iniciado ninguna estrategia en este sentido. Por qué? Porque se apoyan en otros topoi, de clase y de género, que tienen más "peso" a la hora de definir sus trayectorias; pero, además, asimilan el topos dominante de la formación y lo integran como discurso propio, justificando su posición subordinada en el mercado de trabajo. Haciendo un juego de palabras: el hecho de que no se haga algo que se cree y es factible no sólo significa que se creen otras cosas, sino que aquello que se cree y no se ha hecho refuerza la justificación de lo que sí se ha hecho.
} 
Brewer, W. F.; Nakamura, G. V. (1984). "The nature and functions of schemas". A: Handbook of Social Cognition, comp. per R. S. Wyer y T. K. Srull, vol. 1. Hillsdale: Erlbaum.

Carley, K. (1993). "Coding choices for textual analysis: a comparision of content analyisis and map analysis". Sociological Methodology, núm. 23: 77-126.

- (1997). "Network text analysis: The network position of concepts", en Carl W. Roberts (ed.): Text Analysis for the Social Sciences: Methods for Drawing Statistical Inferences from Texts and Transcripts. Mahwah, New Jersey: Lawrence Erlbaum.

Cicourel, A. (1989). "Texto y contexto: aspectos cognitivos, lingüísticos y organizativos de la investigación de campo en las negociaciones internacionales". Papers, Revista de Sociologia, núm. 31: 33-58.

Cicourel, A.; Carley, K. (1990). "The coder of narrative as expert: the ecological validity of coding practices". Ponència presentada a l'Annual Meeting of the American Anthopological Association.

Corman, S. R.; Kuhn, T.; McPhee, R. D.; Dooley, K. J. (2002). "Studying complex discursive systems: Centering resonance analysis of communication". Human Communication Research, 28(2): 157-206.

Fairclough, N. (1989). Language and Power. Londres: Longman.

Freeman, L. C. (1979). "Centrality in social networks: conceptional clarification". Social Networks, núm. 1: 215-39.

Goffman, E. (1974). Frame Analysis: an Essay on the Organization of Experience. Boston: Northeastern University Press., 1986.

Grice, P. (1975). "Logic and conversation". A: Syntax and Semantic. Vol. 3. Speech Acts, ed. per P. Cole y R. Morgan. Nova York: Academic Press.

Holland, D.; Quinn, N. (1997). "Culture and cognition". A: Cultural Models in Language and Thought, comp. per D. Holland y N. Quinn. Cambridge: Cambridge University Press.

J ohnson-Laird, P. N. (1981). Modelos mentales en ciencia cognitiva. A: Perspectivas de la ciencia cognitiva, ed. per D. A. Norman: Barcelona: Paidós, 1987.

Linde, C. (1997). "Explanatory systems in oral life stories". A: Cultural Models in Language and Thought, comp. per D. Holland y N. Quinn. Cambridge: Cambridge University Press.

Lozares, C. (1996). "La teoría de las redes sociales". Papers, Revista de Sociologia, núm. 48: 103-26.

- "El discurs reticular, més enllà de la classificació". Revista Catalana de Sociologia, 11: 183-9.

Lozares, C.; Martí, J.; Verd, J. M. (1996). "Análisis reticular del discurso". Bellaterra: Univ. Autònoma de Barcelona, Departament de Sociologia, Grup d'Estudis Sociològics sobre la Vida Quotidiana i el Treball (QUIT).

Lozares, C.; Verd, J.M., Martí, J.; López Roldán, P. (2003). "Relaciones, redes y discurso: revisión y propuestas en torno al análisis reticular de datos textuales". Revista Española de Investigaciones Sociológicas, no 101/03, págs. 175-200.

Martí, J. (2000). Formació i ocupació en el discurs dels treballadors. Una proposta metodològica. Bellaterra: UAB. Tesis doctoral.

Moeschler, J. \& A. Reboul. (1994). Dictionnaire encyclopédique de pragmatique. Paris: Seuil. 
Portolés, J. (1998). "La teoría de la argumentación en la lengua y los marcadores del discurso". A: Los marcadores del discurso: teoría y análisis, coord. per M. A. Zorraquino y E. Montolío. Madrid: Arco Libros.

Puente, A. (1998). Cognición y aprendizaje. Madrid: Pirámide.

QUIT [Grup d'Estudis Sociològics sobre la Vida Quotidiana i el Treball] (2000). ¿Sirve la formación para el empleo? Madrid: Consejo Económico y Social.

Schank, R. C. (1982). Dynamic Memory: a Theory of Reminding and Learning in Computers and People. Cambridge: Cambridge University Press.

Schank, R. C.; Abelson, R. P. (1977). Guiones, planes, metas y entendimiento. Barcelona: Paidós, 1987.

Searle, J. (1984). Mentes, cerebros y ciencia. Madrid: Cátedra, 1994.

Tordesillas, M. (1994). Prólogo a La argumentación en la lengua, de J. C. Anscombre y O. Ducrot. Madrid: Gredos.

van Dijk, T. A. (1995). "Discourse semantics and ideology". Discourse and Society 6, núm. 2: 243-89.

- (1998). Ideología. Barcelona: Gedisa, 1999.

Wasserman, S.; Faust, K. (1994). Social Network Analysis. Cambridge: University Press. 